\title{
COLLABORATIVE ETHNOGRAPHY FOR INFORMATION SYSTEMS RESEARCH: STUDYING KNOWLEDGE WORK PRACTICES AND DESIGNING SUPPORTIVE INFORMATION SYSTEMS
}

\author{
Ronald Maier \\ University of Innsbruck \\ ronald.maier@uibk.ac.at \\ Stefan Thalmann \\ University of Innsbruck \\ stefan.thalmann@uibk.ac.at
}

\begin{abstract}
Understanding knowledge work and supporting it with information systems (ISs) are challenging tasks. Although there have been numerous studies on knowledge work in varying contexts, many of them are not detailed enough or outdated, particularly with respect to IS support. Knowledge work underwent dramatic changes over recent years, and empirical research studies into how knowledge work is currently performed are scarce. Ethnography would appear to be the most suitable qualitative research method for studying knowledge work, were it not too time-consuming, costly and unfocused for the fast-changing IS domain. Moreover, if we want to ensure the take-up of results from qualitative studies, these need to be transformed into artefacts for the design and engineering of IS requirements. This paper proposes a procedure for the study of knowledge work practices that was adapted from collaborative ethnography, and can be used to inform requirements gathering for, and the design of, IS. The procedure is illustrated with an example of a collaborative ethnographic study which involved seven organisations in four European countries who took part in a large-scale international IS research and development project. The paper also critically discusses the procedure's implementation and its limitations.
\end{abstract}

\section{INTRODUCTION}

Increasing the productivity of knowledge work is a key priority for businesses and organisations in the light of the increase in the share of highly paid knowledge work and of its outputs, with profound impacts on current and future business performance (Drucker 1994; Wolff 2005). Knowledge work takes place in complex social settings. Understanding it and turning the insights gained into artefacts informing IS design are challenging tasks for requirements engineers and designers of ISs. Conversely, not understanding its processes and the (social) conditions of its performance can constitute a major obstacle to successful IS development. Many projects that focused only on technical issues were unsuccessful, since they did not sufficiently take into account the human and organisational dimensions, or the social context of work environments (Al-Karaghouli et al. 2005; Hughes et al. 1994).

Yet, interpretive research is capable of providing deep insights into human thought and action in social and organisational contexts (Walsham 2006). Applied to the study of IS phenomena, and using social constructs (Klein et al. 1999), such an approach would offer a distinct advantage, particularly when the insights can be transformed into the design of IT artefacts (Hevner et al. 2004). Thus, we 
argue that there is a high potential for applying interpretive research methods to design science activities. This can be undertaken by IS academics or by IS practitioners, and is particularly suited to research and development projects which aim to develop IS in complex social settings. Ethnography would appear to be the most appropriate qualitative research methodology for studying knowledge work. However, it is too time-consuming, costly and unfocused for the fast changing IS domain. Moreover, to ensure the take-up of qualitative research results, these need to be transformed into artefacts for the design and engineering of IS requirements. Developing concepts and theories in a behavioural science-based research paradigm differs from developing design artefacts in a design science-based paradigm and insights from one site are not easily transferable to the other.

This paper explores the suitability of a modified ethnography in a large-scale collaborative setting for IS design. The research aimed to answer the question, "How can a modified approach to ethnography be applied in a large-scale collaborative setting to inform IS design?" The paper's distinct contribution is a procedure for the coordination of a collaborative team of ethnographers investigating different social settings, with a common overarching design and research purpose. The aims of this paper are, (1) to present a procedure, based on collaborative ethnography, tailored to the study of knowledge work practices and with a view to enabling requirements gathering and design of IS, and (2) to illustrate the procedure with a collaborative ethnographic study performed in a large-scale IS research and development project. We discuss a large number of recommendations from the literature. We elaborate on the issue of coordination mechanisms that aim to combine the flexibility of an ethnographic approach with a more structured and focused approach necessary for obtaining the type of results that can be transformed into design artefacts. The following section discusses related work and the theoretical background. We then present the procedure step-by-step, including the experiences gained from its application, reflect on the procedure, its implementation and limitations, before we conclude the paper and provide an outlook to further research.

\section{LITERATURE REVIEW}

This section positions our approach from a methodological perspective as bridging interpretive, empirical research with design science research. It then addresses knowledge work practices as objects of investigation, ethnography as an approach to the study of knowledge work practices, and finally argues for a new approach, which combines rapid and collaborative applications of ethnography.

\section{Bridging interpretive and design science research}

There are complex relationships between developing concepts and theories in a behavioural sciencebased research paradigm and developing design artefacts in a design science-based research paradigm. Interpretive research has become more important (Walsham 2006) due to its perceived advantages with respect to providing deep insights into human thought and action in social and organisational contexts. This applies especially to the study of IS phenomena through social constructions such as language, consciousness, shared meanings, documents, tools, and other artefacts (Klein et al. 1999), where meanings are at the centre of the enquiry. The relevance of IS research is directly related to its applicability in designing IT artefacts (Hevner et al. 2004). Empirically-based IS concepts and theories should be easy to implement, able to synthesise an existing body of research or stimulate critical thinking among IS practitioners (Benbasat et al. 1999). Thus, we argue that there is high potential in applying interpretive research methods in design science activities, by IS academics as well as IS practitioners, and particularly in cases when research and development projects aim to develop IS to be used in complex social settings. 
Due to its inherently innovative nature, IT is constantly evolving and IT artefacts are used in emergent application areas where there have not been any empirically-tested concepts and theories (Markus et al. 2002). Here, IT artefacts are first created and used before theories are developed. The theories focus on the impact of applying these IT artefacts to human problem solving and organisational capabilities (Hevner et al. 2004). IT artefacts comprise not only software and hardware, but also concepts and models that represent the problem domain as-is or as-intended. Methods such as simulations, experiments or field studies help in the practice of design. Contributions of design science research comprise design artefacts for solving an identified business need, evaluated constructs, models, methods and instantiations (Hevner et al. 2004) adding to the knowledge base as well as to the methodology.

Concretely, by capturing current work practices of people performing knowledge work we have developed design artefacts that contribute, respectively, to the design of IT support for knowledge work, and to the knowledge base of the IS community. However, in our view, our most important contribution is to methodology, i.e. proposing a procedure for IS design employing modified collaborative ethnography. This methodology is intended to support collaborative research and development projects targeting so-called "wicked problems" (Rittel et al. 1973). These are characterised by unstable requirements and constraints, complex interactions among subcomponents of problem and solution as well as goals that only emerge during design. Research and development projects thus need flexibility in the design processes and artefacts as well as broadening the inherent engineering focus in design science research to a constructivist, interpretive stance.

\section{Knowledge work practices}

The concept of knowledge work(er), coined by Peter Drucker, as well as the concept of Penrose rents, developed by Edith Penrose in her analysis of economic benefits that organisations can get due to their superior idiosyncratic positions have been around for more than 50 years (Drucker 1959; Penrose 1959). Knowledge work in organisations refers to, among other things, creatively solving illstructured problems in complex domains with a high degree of variety and exceptions. It requires the creation, acquisition, application and distribution of knowledge. Inputs and outputs of knowledge work consist primarily of data and information and it concerns mainly organisations with a high share of highly skilled employees. In some industry sectors, knowledge work organisations are characterised by a high number of patents, or by the centrality of customer knowledge, of communications and a high degree of information needs (Alvesson 2004; Hayes 2001; Kelloway et al. 2000; Schultze 2003; Starbuck 1992). The term knowledge work is used in a variety of ways in the literature and can refer to (1) professions, i.e. occupations or job positions that are classified into "knowledge workers" and "non-knowledge workers", the latter being routine or manual workers, (2) group characteristics, i.e. referring to experts' work and thus defining a group of individuals who share certain characteristics appertaining to the nature of their work, and (3) activities or behaviour, i.e. knowledge work is not restricted to a certain class or group of employees, but allows a focus on commonalities concerning work practices and places across professions and positions (Kelloway et al. 2000). We refer to knowledge work here in the latter, activity-oriented sense of knowledge work practices. People performing knowledge work are often highly competent, committed and selforganised. Yet, in order to be productive and effective, they require an organisational environment facilitated by ISs enabling them to jointly apply their competencies in a way that, in Penrose's terms, moves organisations as a whole into superior idiosyncratic positions or retains them there.

Knowledge work is prevalent in high-tech industries and expert-driven organisations such as in professional services, engineering or pharmaceutical sectors (Graham et al. 2004). However, we argue that knowledge work is sufficiently similar across occupations and industries to allow designing generic instruments to foster knowledge work. According to our results, people performing knowledge work typically rely mostly on standard IS tools, such as office, communication and 
collaboration systems, and the development of IS for knowledge work is mostly delegated to external IS companies which aim at offering general purpose solutions targeting a diverse user base.

Aided by the widespread use of advanced collaborative IS, knowledge work has changed substantially in recent years. Whilst knowledge management has seen an abundance of concepts, models, methods, tools and systems, mostly in the 1990s (Alavi et al. 2001; Blackler 1995; Brown et al. 1998; Davenport 1998; Nonaka 1991; Wiig 1993; Zack 1999), many of which have failed to achieve their goals (Bishop et al. 2008), studies on how knowledge work is currently performed are scarce, not detailed enough or outdated particularly with respect to IS support. There were quite a few studies in the 80s and 90s on how computers impacted on the behaviour of groups (Dennis 1996; Suchman 1987). Since then, there has been a general shortage of workplace studies identified for quite some time in organisation science (Barley et al. 2001), with particular needs highlighted in relation to studies on the support of knowledge work through IS.

One important factor for the scarcity of research in this area lies in the difficulty of studying knowledge due to its metaphysical nature (Schultze 2000). Blackler (1993), therefore, recommends that knowledge work be studied by focusing on work practices. This strong empirical focus on what people do, rather than on what they know, and, one might add, why and how they do what they do, calls for a much more thorough investigation.

\section{ETHNOGRAPHY}

Ethnographic research was developed to investigate new cultures and social settings and is well established in anthropology and social sciences as the "art and science of describing a group or culture" (Fetterman 1999). The first ethnographers described exotic cultures in remote islands, presented in form of monographs (Malinowski 1922). Even to this day, ethnography seeks immersion or active participation in social settings to understand why and how things happen (Jordan 1996).

An ethnographer aims to become a member of the community by working with people in their natural environments, typically for long periods of time (Fetterman 1999), whereas a conventional researcher never becomes a member in the same way (Emmerson et al. 1995). Motivations and intentions of participated actions can be observed by the ethnographer (Hammersley et al. 2007). A key success factor is to be open to all kinds of situations rather than restricting one's focus by excluding situations (Fetterman 1999). Theories are developed inductively from the data gathered, rather than proposed and tested. Ethnographers should be aware that it is difficult to ignore prior knowledge and ethnographers are guided by hypotheses and ideas (Atkinson et al. 2007; Orlikowski 1993).

Recently, ethnography has become more popular in disciplines other than anthropology and sociology (Ball et al. 2000; Harper 2000). This is especially true for design activities which focus on users and their thoughts (Sharrock et al. 2004). There have also been a number of studies in the IS field (Harper 2000; Myers et al. 1997; Orlikowski 1991; Suchman 1995). These studies were aimed at developing a thorough understanding of current work practices as a basis for the design of IS support (Simonsen et al. 1997). While traditional software engineering methods often fail to consider crucial aspects of the users' environment, such as the social context of work and organisation, ethnographic research explicitly focuses on the social settings in which the intended system should be used (Hughes et al. 1994; Myers 1999). However, an ethnographic approach tends to be much more in-depth and time consuming than traditional software engineering methods.

\section{Rapid and collaborative ethnography}

Traditional ethnographic studies require between six months to 2 years' fieldwork, ideally studying anything that happens without preoccupations (Fetterman 1999). Modified versions, called rapid 
ethnography (Millen 2000), 'quick and dirty' ethnography (Hughes et al. 1994) or applied ethnography (Ball et al. 2000; Maginn 2007) have been proposed as less time-consuming and more appropriate research approaches for informing IS design (Harper 2000).

The reduced period of fieldwork constitutes the biggest difference with traditional ethnographic approaches (Hughes et al. 1994). This can be achieved by keeping the focus rigorously narrowed down to the specific study's goals (Millen 2000), including by explicitly considering concepts and theories without excluding the openness for the unforeseen (Ball et al. 2000). Thus, the ethnographer does not aim at a complete and detailed understanding of the whole social setting, but rather at an understanding of aspects deemed relevant to the study (Hughes et al. 1994). Here, applying the 'viewpoint analysis' technique enables the definition and selection of such viewpoints (Viller et al. 2000). Furthermore, time may be saved by using a range of different observation techniques and tools (Millen 2000), such as photography, audio and video recordings, to enhance the data collection (Crabtree 2003; Pink 2006); and by working collaboratively and, hence, parallelizing tasks (Millen 2000). However, it should be noted that parallelizing is limited as a certain depth of immersion is required. The most distinguishing feature of ethnographic studies is the use of ethnographic observations (Warne et al. 2005) in the field. Researchers acquire rich data representing the context by immersing themselves in the researched situations. Thereby, work procedures can be observed as they take place in different settings, and questions can be asked if clarification is needed (Warne et al. 2005).

Modified ethnographic studies are usually realised in the case of highly situated work practices and a need for specific support (Plowman et al. 1995). Such studies have been used for a variety of purposes, e.g., to perform complete requirements analyses of engineering processes, (Ball et al. 2000; Weng et al. 2006), to provide additional information to traditional requirements engineering methods, (Bentley et al. 1992; Simonsen et al. 1997; Viller et al. 2000), to investigate the impact or the quality of existing software, (Robinson et al. 2007), or to study real-world phenomena for theory-building, (Maginn 2007; Millen 2000; Tuula et al. 2006).

By modifying the characteristics of traditional ethnography, the boundaries between different qualitative research strategies become blurred. Ethnography and case study can be seen as two independent research strategies on the same level (Creswell 2003; Darke et al. 2002; Visconti 2010; White et al. 2009). However, there are several similarities between case study and ethnography (Klein et al. 1999). The main difference between case studies and ethnographies is the extent to which the researcher immerses into the research setting (Myers 1999). Immersion in this context is the hallmark of ethnography and constitutes an "attempt to understand the way others construe, conceptualise, and understand events, concepts, and categories, in part because these are assumed to influence individuals' behaviour" (Kaplan et al. 1988). In case studies, the primary source of data is the interview, in which respondents answer from their individual perspective. In contrast, ethnographers collect data primarily through observation and participation in natural social settings over a prolonged period of time (Creswell 2003) is essential for ethnography. Ethnography is seen as inward-looking, aiming to uncover the tacit knowledge of a culture or a setting, while case study is seen as outwardlooking, aiming to describe the nature of phenomena through a detailed investigation of individual cases and their contexts (Cohen et al. 2003). Thus, even though some characteristics of ethnography may be modified, such as spending less time in the field, which needs to be acquainted for, e.g., by enhancing data collection or applying a focus on certain aspects to be studied, the primary differentiating characteristic of ethnography needs to be retained, i.e. allowing enough time and openness for the researcher to immerse into the studied social setting.

Compared with surveys, ethnographic studies are usually performed by a single researcher and limited to the practices, social settings and culture of only one organisation, or in the case of medium to large organisations, confined to the study of a small section of the organisation. This is commonly 
criticised (Myers 1999) and limits applicability of the results for design activities that aim at supporting users across departments, organisations or even industry sectors. This calls for a collaborative approach among ethnographers to address this limitation.

Furthermore, collaboration between ethnographers can increase the quality of a study and of its results by bringing in different perspectives (Buford-May et al. 2001). Collaborative ethnographic studies deliberately and explicitly emphasise collaboration in all its aspects, from conceptualisation, to fieldwork, analysis and discussion (Lassiter 2005). However, the goals, concepts and theories held by the participating ethnographers need to be coordinated in order to get comparable results.

Collaboration during IS design is not new, however, and a variety of target groups have been investigated, including the collaboration between teams of software engineers (Whitehead 2007), between stakeholders and designers (Luna-Reyes et al. 2008), user groups and designers (Wu et al. 2003) or between software users and designers (Kendall et al. 1986). Furthermore, methods of data collection imported from social sciences have been applied in IS research, such as interviews (Walsham 1995), observations (Wu et al. 2003) or folklore technique (Kendall et al. 1986). The distinct contribution this paper makes is by proposing a procedure for the coordination of a collaborative team of ethnographers investigating different social settings, with a common overarching design and research aim.

\section{PROCEDURE}

This section describes the procedure we propose for modified collaborative ethnography based on related work that helped in its elaboration, step by step. Each step is illustrated with a case described as follows, that also allows us to reflect on the lessons learned.

\section{Case description}

In our large-scale research and development project MATURE, funded by the European Commission under its Framework Programme 7 (www.mature-ip.eu), a collaborative ethnographic study was realised by 18 ethnographers who participated in the daily work lives of 31 employees predominantly performing knowledge work. The study comprised seven instances, i.e. seven organisations in four European countries. The sample was designed to include a wide range of organisations representing a mix of contexts and characteristics deemed to influence knowledge work, i.e. size, sector, IT intensity and country (see Table 1).

\begin{tabular}{|l|l|l|l|l|}
\hline organisation & size (employees) & sector & IT intensity & country \\
\hline I & large $(20,000)$ & telecommunication & high & Switzerland \\
\hline II & large $(4,000)$ & health care & medium & Germany \\
\hline III & large $(1,000)$ & professional services & medium & United \\
\hline IV & large $(400)$ & IT services & high & Germany \\
\hline V & large $(300)$ & professional services & medium & United \\
\hline VI & medium $(130)$ & IT franchising & high & Germany \\
\hline VII & small $(30)$ & consulting services & medium & Spain \\
\hline
\end{tabular}

Table 1: Characterisation of the sample

The authors took a lead role in the study, by undertaking its design, running a pilot, coordinating study instances and leading the data collection, analysis, reflection and writing-up of results. 
Furthermore, the authors ensured the take-up in the project's software development activities which included requirements analysis, design activities and evaluation. The time frame for the study was limited, partly because some organisations were not willing to accept researchers participating in daily activities for more than two weeks. Allowing researchers to participate in and investigate employees' daily work practices requires a substantial amount of trust. Hence, we relied on the voluntary participation of organizations with whom the researchers had some kind of prior relationship, whether through personal contacts within the organisation or jointly performed project work. Furthermore, the researcher's existing knowledge of the organisations made it easier to assess and select the appropriate organisational units to be visited and their suitability for the study. Additionally, their knowledge and their existing personal relationships sped up the immersion into the organisational contexts. Immersion was further aided by the fact that ethnographers and subjects had similar professional and cultural backgrounds. Ethnographers were familiar with the aspects of knowledge work to be studied from their own professional experience as knowledge workers. However, in order to mitigate a potential bias in this selection, we included two organisations with whom we had no such prior relationships. Within the organisational settings, we studied knowledgeintensive processes, such as product development or customer relationship management and employees who were primarily engaged in knowledge work, such as IT consultants or project managers.

\section{Phase 1: Definition}

First of all, the study team needs to decide whether the design problem it targets with its research and development project is indeed "wicked" (Rittel et al. 1973), i.e. characterised by unstable requirements and constraints as well as complex interactions among subcomponents of problem and solution among others. If this is the case, the procedure will be beneficial, otherwise more traditional procedures and methods for requirements engineering and design would probably be more costeffective. Fundamental to our procedure for collaborative ethnography is that several researchers use the same approach to answer a joint research question (Lassiter 2005). However, they perform fieldwork individually in one unique social setting with the collective arrangement in mind. Each individual study performed by any one ethnographer can be seen as constituting a distinct ethnographic study in its own right, as well as one part of a collaborative study. Researchers bring their own personal perspectives to the study, reflecting their different professional backgrounds, and thus describe and highlight different details and aspects. Thus, collaborative ethnography needs to provide a process for combining these perspectives, into a broader view on the setting (Buford-May et al. 2001), particularly if study instances are performed in different social settings.

Ethnographic studies cannot be sped up without modifying the corresponding procedure (Crabtree 2003). In order to ensure the effective use of limited time, the ethnographer needs to narrow down the study's focus to objectives relevant to design activities (Ball et al. 2000; Hughes et al. 1994; Millen 2000) and only capture the main characteristics of the setting, i.e. aspects related to concept development and prospective design tasks.

Focusing parts of the setting requires the coordination of ethnographers. Guidelines describing general procedures in terms of do's and don'ts are useful (Hammersley et al. 2007). Due to the uniqueness of each social setting and the situational character of ethnography, it is impractical to develop detailed guidelines ex ante. Evaluating informant-expressed needs and considering them when developing guidelines can be one way of coordination before performing study instances (Lassiter 2005). Due to the restricted focus and the shortened time, modified ethnography also benefits from a strong theoretical framework guiding the fieldwork (Ball et al. 2000) or it risks leaving out important aspects. Guidelines for fieldwork therefore should contain descriptions of the study's goals and areas of focus that are based on established concepts and theories. 
In collaborative ethnographic studies, it is necessary to agree on ways to share experiences and data (Gordon et al. 2006).Ethnographers should communicate about and agree on goals and focus areas to ensure common understanding. This turned out as very effective for a distributed group of ethnographers having different professional backgrounds. Goals and areas of focus need to be balanced, i.e. on the one hand sufficiently general to allow elaborating on interesting individual aspects in study instances and, on the other hand, sufficiently detailed to guide fieldwork effectively. More heterogeneous professional backgrounds and interests of ethnographers, and more diverse studied social settings, require more generic guidelines. Thus, coordination is aided by shared resources (Malone et al. 1994). These shared resources can be guidelines and later on design artefacts used as boundary objects (Leigh Star et al. 1989). An experienced study coordinator (designated to the group) will be able to facilitate a dispersed team, to broker experiences between team members and will have the authority to resolve any issues or disagreements. The archetype of a study coordinator is adopted from medical studies, in which coordinators commonly accompany whole studies, develop forms and guidelines for coordination and serve as the main contact person (Davis et al. 2002).

Becoming familiar with the social setting can take considerable time during which the ethnographer's capacity for absorption is reduced (Millen 2000). In order to minimise this time, ethnographers ideally are already familiar with the domain in which studied individuals act in order to make it easier to understand their activities (Ball et al. 2000). Studying documented information about the setting ahead of the actual study also sped up the familiarisation process in our study. Knowing organizations, workplaces or social settings from previous interactions significantly reduces familiarisation with the study milieu, as social relationships are already established. Meetings preceding the study can be useful to become familiar with individuals and their context early-on (Ball et al. 2000). Whilst prior knowledge of organisations speeds up time needed for immersion, potential bias in the procedure should be balanced. Some authors argue that it is generally very difficult or even impossible to perform an unbiased procedure and to ignore common known knowledge, no matter whether previous relationships exist or not (Glaser et al. 1979; Gläser et al. 2006; Walsham 2006). The researcher can hardly avoid to be guided by hypotheses and ideas, which motivate his fieldwork and the selection of the social setting to be studied. Instead of dismissing the theoretical background and existing knowledge about the social context, the ethnographer should be aware of them (Atkinson et al. 2007; Fetterman 2010): the willingness to reflect on and revise initial assumptions and theories is considered crucial in this regard (Walsham 2006). Joint reflection of ethnographers can here be exploited as one of the advantages of collaborative ethnography, particularly if they investigate related settings, e.g., within one organisation. Taking the previous deliberations into account, the following procedure is suggested:

- Base the definition of goals and focus areas on a strong theoretical framework.

- Define the role of a study coordinator and assign it to one ethnographer.

- Analyse artefacts which provide information about the research setting.

- Meet prospective participants before the start of the study.

- Define conditions, such as the ethnographers and the social settings.

- Communicate the study outline to all ethnographers, and ensure that they share the same understanding.

- Establish acceptance of planned activities by the organisations studied.

Applying this procedure, our study had two closely related aims. The first was to investigate knowledge workplaces using practices of knowledge work as a guiding theme. The second aim was to gather requirements and support the design of an IS supporting chained practices of knowledge work aimed at developing collective knowledge in organisations. 
We negotiated the organizational framework within the project consortium. The first and most crucial point was to define the goals and the focus areas of the study. The authors made a proposal detailing and agreeing the project goals for the study. Five overarching focus areas could be developed and refined in a collaborative procedure involving the other project partners. All ideas gathered within this procedure were consolidated and agreed at one face-to-face meeting with all ethnographers. The first focus area was knowledge maturing and the stages in this process. The second was the knowledge routines and knowledge processes (Davenport et al. 1996) and their ICT support. The third focus was the usage of knowledge in specific situations or knowledge-oriented actions (Ellis 1993; Knights et al. 1993; Schultze 2000). The fourth focus was the motivation of knowledge workers during interaction with knowledge. The last focus was the structure of knowledge in organisations. Thereby, formal structures such as folder hierarchies were deemed particularly important. Between 2 and 6 more detailed questions, such as: "in which situations do people give up their privacy?" or "are there any policies or rules for structuring knowledge?" were used to describe each focus point in more detail.

Building on these focus points, the authors proposed a study design. The proposal was discussed once more, slightly adapted and finally approved in another face-to-face meeting with all ethnographers. In the same meeting, organizations which were considered suitable for the study were identified and discussed after each ethnographer team had proposed potential organisations by providing a short presentation on the organisation, its units and processes as well as the intended time schedule. Finally, the project consortium agreed on the organisations and processes within those organisations to be studied on the one hand as well as teams of ethnographers on the other hand.

\section{Phase 2: Pilot study}

The main aim of this phase is to familiarise researchers with phenomenon and context. Using a single case as a pilot study is beneficial if the research is exploratory (Benbasat et al. 1987). In a collaborative setting, the study coordinator should participate in one study instance, ideally in the pilot study. Experiences gathered in the pilot study can be written up as "good practice" (O'Dell et al. 1998) for other ethnographers which successfully sped up the fieldwork in our case. When time is of the essence, the additional effort might outweigh the advantage of lessons learned to be already taken up in the study's further instances, particularly if participating ethnographers already have extensive prior experiences in the study's domain and social settings. The following procedure is suggested:

- Perform a pilot study.

- Transfer lessons learned to ethnographers.

- Identify "good practices", refine goals, focus areas and guidelines.

- Communicate the refined guidelines to ethnographers, and ensure that they agree with them.

The pilot study was performed by the authors. Due to the success of the pilot study, the procedure of the study was applied to the other study instances. The pilot study took five weeks in total, of which two weeks were used for fieldwork, i.e. ethnographic observations and immersion (see phase 3 fieldwork for details of the procedure). Only some minor changes were made to the focus areas and guidelines while the goals stayed the same. Thus, the pilot study is fully comparable with the other study instances.

Our familiarity with the domain and the trusted relationship with the participants proved to be highly beneficial for an effective use of the scarce time and sped up the immersion into the work context. The trusted relationship developed through a previous positive experience of a joint project resulted in strong management support for our study and an unobstructed, open work atmosphere with participants.

The pilot study allowed the authors to transfer experiences collected on the procedure early on to the other ethnographers in a workshop-style meeting. The authors reported their experiences of the study 
and elaborated on their experiences in the form of anecdotes and stories. The procedure of the pilot study and experiences from the fieldwork provided the focus for this meeting, not (preliminary) results and reflections on them. The anecdotes and stories were presented in a sequence of distinct phases of the fieldwork. In the workshop, they were jointly reflected as lessons learned, taking on board previous experiences of all participating ethnographers. Examples of questions that were targeted here and for which general guidelines have been agreed on were: how to address management, how to conduct the first meeting with the study participants, how to raise awareness for the study's focus areas, how to conduct the first day of the study, how to handle breaks, when to write field notes, how to determine the appropriate level of detail of field notes, how to split the time spent with the individuals studied and spent on isolated reflection, how to handle the uninterrupted observation of work practices, how to discuss self-reporting with studied individuals ex ante and ex post, how to keep contact during the period between site visits or how to perform the post-hoc reflection. The aim was not to provide a rigid set of rules, but to jointly go through the handling of circumstances that each ethnographer was likely to encounter and to reduce the cognitive load required for decisions that need to be taken in the field. In this way, we substantially reduced the distraction from fieldwork.

\section{Phase 3: Fieldwork}

Reducing time for fieldwork can lower sensitivity to longitudinal aspects about practices of knowledge work. The period of fieldwork can be split in order to give participants more time to critically reflect on aspects of knowledge work. Communication channels, such as chat, e-mail or videoconference, can be used to bridge the time in between. This form of communication cannot replace "staying in the field", but it can help ethnographers to stay in contact with participants and to be informed about ongoing activities (Hine 2000). Participants can use templates to report on events related to the study, which requires their awareness for the study's focus areas. Ethnographers need to help create awareness of participants during the initial period of fieldwork. The reported events can then be taken up in the second field visit and discussed with the ethnographers returning to the site.

Technology can be used to collect more data in less time, e.g., photography, audio and video recordings (Millen 2000; Pink 2006). However, recordings require participants' consent, which may considerably impact on their behaviour, and recording devices can only record what is observable and in their focus (DuFon 2002). Data gathered in this way are additional sources which need to be interpreted and linked to the study's context (Pink 2006).

Ethnographic observations are the defining element of the modified approach to ethnography. The researchers immerse themselves into the social setting, take part in a variety of situations and ask questions if needed (Warne et al. 2005). During fieldwork, practitioner-researchers usually reflect on occurrences and activities in the field (Schön 1983). This reflection-in-action should be understood as an iterative process in which ethnographers are guided by an "overarching theory" (Dickover 1994) and construct new theory of the unique case during their practice (Schön 1983). The aim should be to learn more about occurrences in the field and to become aware of new aspects or to ask other questions.

It would seem useful for the study coordinator to attend the start of every study instance in order to gain a direct impression of the instance's setting and to coach ethnographers during initiation. The role should not be misunderstood as aiming to control ethnographers, but to get an impression aiding the later synthesis of results from combined study instances. Too much involvement could also constrain ethnographers and hamper the study instance's initiation. The following procedure is suggested:

- Plan how to involve the study coordinator in instance studies.

- Perform ethnographic fieldwork and create awareness for the study's focus. 
- Support participants in self-reporting and stay in contact with them.

- Perform ethnographic fieldwork and discuss self-reporting.

Due to the time constraints given by some of the participant organisations, time for fieldwork within the study was generally limited to two weeks. The time was divided into two periods of one week each, bridged by a period of two to three weeks for self-reporting. This approach allowed ethnographers to investigate medium and long running work practices at different stages and turned out to be very beneficial. Due to the immersion into work situations and the rich spectrum of gathered data, our data collection approach goes far beyond interviewing. Participating in all aspects of the work, including work process and social life, casual meetings, breaks and also some joint leisure time activities, allowed a very quick and thorough immersion. Furthermore, using directed questioning combined with observation and also self-reporting of work activities lead to a deeper understanding and contextually rich data.

We created a template for the self-description period based on the focus areas and established concepts we had previously agreed, in our case the concept of knowledge stance (Maier 2005). A knowledge stance is a trigger for a knowledge-oriented action in which knowledge is created or reconstructed. It comprises steps which can be supported by IT services and are framed by a defined start occasion and end state used to structure and describe knowledge work situations. The main viewpoints were briefly explained and one short example was given. During the first week of fieldwork, participants were instructed in the use of this template.

Awareness and sensitivity for situations in which participants handle knowledge in a non-routine way were created during the first week of fieldwork as the main focus of self-reporting. Raising awareness of participants about the study's focus areas was beneficial so that they could share their reflection-inaction with ethnographers providing further background information. Thus, we could discuss data collected over a five weeks' period during which participants were engaged in different tasks and project phases. Time spent with participants versus isolated reflection was split so that time with participants was maximized while ethnographers had the time to retreat as and when required. This enabled the researchers to spend most of the working day with participants, including lunch and coffee breaks. Some teams of ethnographers had a separate room at their disposal in their studied organisation where they could meet for isolated or joint reflection which helped reflection-in-action as close to the action as possible, both physically and time-wise.

One of the authors acted as study coordinator and thus attended at least the first day of each study instance in order to accompany participating researchers. The main aim was to provide support for ethnographers, to ensure comparability between instances and to get an overall impression of the settings. The study coordinator accompanied ethnographers without interrupting them and tried to stay in the background. During breaks and at the end of each day, he facilitated a joint reflection on the fieldwork. Participating researchers frequently asked for suggestions on how to handle emerging problems and circumstances. Background knowledge and personal impressions gained during participation proved very useful for coordinating the collaborative data analysis.

\section{Phase 4: Data Analysis}

Field notes are the main artefact for analysing ethnographic data (Ball et al. 2000). Due to its highly contextualised nature, the data can only be analysed effectively by those researchers who performed the fieldwork (Emmerson et al. 1995). Hence, it is not advisable to centralise data analysis in a collaborative study. Field notes should be analysed locally and they can be coordinated by templates (Lewis et al. 1996). This procedure is strongly related to reflection-on-action whereby ethnographers think back on situations in the field, on actions by participants of the study and also on their reflection-in-action (Schön 1983). In a collaborative setting, this reflection can be supported by 
formal "after action reviews" in which groups of researchers jointly reflect on occurrences in the field (Baird et al. 1999). However, reflection-on-action is not performed arbitrarily, but employs a method for qualitative data analysis.

This typically involves coding field notes, i.e. the categorization of field notes into concepts suggested by the field data (Agar 1980). In traditional ethnography, codes are ideally developed in a purely inductive way without previous deliberations. However, even in case of only one ethnographer and an extended period of time, it is considered difficult to ignore common knowledge and prior deliberations (Glaser et al. 1967). One approach bridging both perspectives is the axial coding in which inductively developed categories are linked to theories in order to select and refine them (Strauss et al. 1990). Due to its undirected nature, the purely inductive procedure can be considerably time-consuming which seems inefficient in a collaborative setting. Hence, initial codes or at least categories, should be developed based on precisely defined concepts and the focus areas agreed between researchers (Lewis et al. 1996). Openness requested in inductive approaches is ensured by adjusting and extending initial categories flexibly if deemed necessary in a study instance (Patton 2002).

Results from study instances need to be consolidated. This can be realised centrally, collaboratively or in a combination of both. In any case, a coordinating role seems to be effective, and personal experiences about settings of individual study instances gathered by the study coordinator invaluable. There is an important issue here about when ethnographers should let go of their private data and findings. At a certain point in time results need to be transferred to designers who should benefit from the broadened perspective of the collaborative study without having to deal with all the specifics of each study instance. We suggest a gradual approach beginning with individual data analysis, sharing results with other ethnographers early on, negotiating how to document findings, making the context as explicit as necessary for ethnographers to compare their findings and interpreting these collaboratively. This leads to a consolidated set of results rather than to a collection of isolated results.

Several authors propose principles or criteria for validating qualitative research (Horsburgh 2003; Klein et al. 1999; Lincoln 1995; Patton 2002; Seale 1999). These criteria cannot be applied in a mechanistic way and need to be adapted to each study individually (Klein et al. 1999). However, these principles can be seen as basic rules of research, forming the philosophical base of interpretative research and can hence also be applied to the study design of collaborative ethnography. The following procedure is suggested:

- Define templates to guide joint analysis of field notes.

- Develop initial codes or categories.

- Share results early on and make context explicit.

- Consolidate individual findings into a collaborative set of findings.

- Use one set of principles for evaluating interpretative field studies.

A workshop for all ethnographers was set up to share experiences from fieldwork as well as to agree on the procedure for data analysis. Each team of ethnographers presented their study to get an overview of all study instances. While the other teams of ethnographers performed their fieldwork, the authors already started with the data analysis and developed a proposal for a collaborative procedure. Templates were developed that contained general conditions of the intended description of results without restricting specific results. These templates were refined in a multi-step process in order to take on board specific experiences by study instances that might be of interest to all instances and guided data analysis. Researchers were requested to identify further relevant aspects that should be considered in all study instances during this multistep negotiation process. Furthermore, a timeline coordinating the integration of the results of the individual data analyses was proposed, discussed and approved. This illustrates overlapping phases, an approach we took to further improve timeefficiency. The study coordinator continuously stayed one step ahead concerning the study instance in 
which he participated, preparing the next phase while still engaged in coordinating the previous phase.

Coding was supported by a structural model of situations covering the six dimensions when, who, why, where, what and how. Characteristics of the former four generic dimensions were developed inductively, while characteristics of the latter two specific dimensions were developed with an informed inductive procedure (Patton 2002). This resulted in a set of sequences of knowledge routines with specific demands regarding ICT support, a list of indicators for assessing their performance and a typology of knowledge work situations and knowledge elements. Together with identified barriers and enablers, these results were used to detail our "wicked problem"(Rittel et al. 1973), especially in the area of ICT support.The principles proposed by (Klein et al. 1999) for evaluating interpretative research were used to ensure the quality of our study. The set of principles turned out to be useful for reflecting on the procedures used for data collection and for data analysis. The results were turned into proposals how to develop, structure, describe and visualise implications for design activities in the subsequent phase. Guidelines for reflections in local teams as well as the agenda for the entire project meeting were developed. The design principles and the implications for the study on the local team of ethnographers as well as on the entire project group are depicted in Table 2.

\begin{tabular}{|l|l|l|}
\hline $\begin{array}{l}\text { principle } \\
\text { according to } \\
\text { (Klein et al. 1999) }\end{array}$ & $\begin{array}{l}\text { measures taken in the } \\
\text { individual social setting and } \\
\text { the study instance }\end{array}$ & $\begin{array}{l}\text { measures taken in the } \\
\text { collaborative study as a whole }\end{array}$ \\
\hline $\begin{array}{l}\text { principle of the } \\
\text { hermeneutic circle }\end{array}$ & $\begin{array}{l}\text { local social phenomena were } \\
\text { reflected in regard to the entire } \\
\text { organisation }\end{array}$ & $\begin{array}{l}\text { consolidated interpretations were } \\
\text { reflected regarding the study's } \\
\text { aims }\end{array}$ \\
\hline $\begin{array}{l}\text { principle of } \\
\text { contextualisation }\end{array}$ & $\begin{array}{l}\text { historical background with the } \\
\text { social setting was disclosed and } \\
\text { discussed }\end{array}$ & $\begin{array}{l}\text { research settings were presented } \\
\text { and their suitability and } \\
\text { limitations discussed }\end{array}$ \\
\hline $\begin{array}{l}\text { principle of } \\
\text { interaction }\end{array}$ & $\begin{array}{l}\text { socializing to the views of the } \\
\text { participants was discussed }\end{array}$ & $\begin{array}{l}\text { short summary on the interaction } \\
\text { was provided and jointly reflected } \\
\text { upon }\end{array}$ \\
\hline $\begin{array}{l}\text { principle of } \\
\text { abstraction and } \\
\text { generalisation }\end{array}$ & $\begin{array}{l}\text { design artefacts were created, } \\
\text { discussed and refined }\end{array}$ & $\begin{array}{l}\text { initial coding scheme was based } \\
\text { on concepts known from the } \\
\text { literature }\end{array}$ \\
\hline $\begin{array}{l}\text { principle of } \\
\text { dialogical } \\
\text { reasoning }\end{array}$ & $\begin{array}{l}\text { the coding scheme was } \\
\text { reviewed and revised after the } \\
\text { first coding }\end{array}$ & $\begin{array}{l}\text { the coding scheme was jointly } \\
\text { reviewed after individual coding }\end{array}$ \\
\hline $\begin{array}{l}\text { principle of } \\
\text { multiple } \\
\text { interpretations }\end{array}$ & $\begin{array}{l}\text { individual interpretations were } \\
\text { jointly reflected during } \\
\text { artefact creation }\end{array}$ & $\begin{array}{l}\text { consolidated interpretations per } \\
\text { study instance were jointly } \\
\text { reflected after fieldwork, after } \\
\text { coding and after artefact creation }\end{array}$ \\
\hline $\begin{array}{l}\text { principle of } \\
\text { suspicion } \\
\text { were called back }\end{array}$ & $\begin{array}{l}\text { consolidated interpretations per } \\
\text { study instance were challenged by } \\
\text { people not engaged in the } \\
\text { fieldwork }\end{array}$ \\
\hline
\end{tabular}

Table 2: Discussion of evaluation principles 


\section{Phase 5: Artefact creation}

The take-up of results in design activities raises issues of how to communicate findings from ethnographic studies to design teams (Hughes et al. 1994) and to transform results into parts digestible by designers (Crabtree 2003; Viller et al. 2000). Codes and categories are too abstract and intangible. They need to be translated into design artefacts useful for designers, e.g., requirements, use cases or process models, guided by the purpose of design (Lewis et al. 1996). As there are farreaching decisions to be taken, intermediate steps might help to reduce the complexity of moving between levels of abstraction and also to provide boundary objects that ethnographers and designers can both relate to (Chang et al. 2008). Artefacts known from object-oriented analysis, such as case diagrams or sequence diagrams are suitable if existing systems are in the focus of research (Viller et al. 2000). However, richly described artefacts, such as personas, i.e. fictitious, concrete representations of target users which describe what they want to accomplish in regard to a design task (Pruitt et al. 2006), are beneficial in case of designing a tool for work practices that so far have not been supported.

Modified versions of ethnography are goal-oriented. Fieldwork and data analyses are directed towards creating such artefacts, hence artefacts can be expected to inform IS design. Ethnographers act as communicative agents between users and designers (Crabtree 2003). Study results can be used for scoping and prioritising design activities, for designing IS that target critical social settings and for evaluation (Lewis et al. 1996).

As a process iterating with data analysis, artefacts should also be created collaboratively (Lewis et al. 1996). Furthermore, the study coordinator should guide the process to ensure the comparability of artefacts that can be understood by designers. The appropriateness of design artefacts must be rigorously shown via well-executed evaluation methods (Hevner et al. 2004). The evaluation of the created artefacts provides new insights which can be used to improve the quality of the product as well as the design process, i.e. the study design (Hevner et al. 2004). The second point demands a reflection of the data analysis and its results in particular. The fit of the IS created on the basis of the design artefacts to the tasks of the employees can be evaluated, e.g., by assessing the task technology fit (Goodhue et al. 1995), the effectiveness of the IS in general (Hamilton et al. 1981) or the IS success (DeLone et al. 2003). Ultimately, the IS needs to be adopted, i.e. an organization decides to use the IS (Rogers 1995), it needs to be accepted, i.e. a user decides voluntarily to use the IS (Dillon et al. 1996) and assimilated, i.e. the IS-based solution diffuses into organizational work processes and corresponding daily activities (Chatterjee et al. 2002). The following procedure is suggested:

- Create artefacts suitable for designers.

- Ensure findings are used to inform design and to evaluate design artefacts.

- Apply suitable evaluation methods to check the appropriateness of artefacts.

Results and insights gained from the study were shared in numerous meetings and teleconferences with designers to jointly refine them into design artefacts. The procedure was initiated by a modelling workshop. The authors prepared design artefacts such as personas or process models in advance and presented them to the consortium. The suitability and ideas for revision were discussed and a time schedule for the artefact creation was agreed. Participating researchers were requested to search for additional design artefacts and encouraged to create proposed artefacts out of their field data.

This initiation of a process for creating different kinds of design artefacts was very fruitful and resulted in a large number of design artefacts. These reflect the agreed consolidated results of the data analysis as well as the individual experiences of ethnographers. Hence, artefacts needed to be consolidated. Due to the fact that the artefacts should be used for the design activities, a joint meeting involving designers and ethnographers seemed useful. Design artefacts of the same type were categorized and grouped as part of preparing for this workshop. This initial grouping provided a 
beneficial starting point for the break-out group discussions in the meeting. The goal of each breakout group was to select and consolidate the most suitable design artefacts out of a given set. Thus, the design artefacts could be reduced to a manageable number. Furthermore, a hand-over of design artefacts from ethnographers to designers took place. The necessary knowledge transfer was aided by the fact that both groups collaboratively discussed the design artefacts and by the ethnographers and designers constituting overlapping groups.

Finally, chains of knowledge practices were described with the help of process models and enriched by further model types and elements, i.e. goals, indicators, personas, knowledge elements, services and barriers. Personas formed important communication media in the project and helped consolidate findings and guide decisions about ISs. Templates had been used during data analysis, such as a table of characteristics of personas or criteria for identifying processes to be supported by the intended system.

Evaluation scenarios were developed from the gathered requirements and used in design, prototyping and later on in testing. Software demonstrators targeting the support of the identified knowledge processes were created and evaluated. The evaluation of these demonstrators was performed by researchers who had already participated in the fieldwork, and the results of the evaluation were used to refine the design artefacts and to justify the applicability of concepts built into the data analysis, e.g., instantiations of indicators for assessing services supporting knowledge work.

A second stream of evaluation was performed in regard to the theoretical results of the study. Several concepts and models that had been created or refined in this collaborative ethnographic study were empirically tested with the help of semi-structured interviews with a much larger sample of organisations in a follow-up study. Examples are the importance, state-of-support and success of performing the identified knowledge practices, the importance of barriers and ways how to overcome them, supporting IT services and the ways in which organisations have successfully appropriated technology in support of knowledge practices.

\section{DISCUSSION AND LIMITATIONS}

Figure 1 summarises the five phases of our procedure which are similar to the common research procedure for qualitative research (Patton 2002) and also describes major inputs, outputs and activities of the study coordinator in each phase. In the following, we discuss the specifics of the proposed procedure. Phases are performed in sequence but overlapping with each other. The output of the preceding phase is used as input for the next phase. Phases 1 and 2 as well as phases 4 and 5 are performed iteratively so that the results of the pilot study are used to reflect the definition of the study design, and the artefact creation influences the data analysis. The latter materializes an important specific characteristic of this procedure, namely that the study's goals are explicitly targeted at the design of an (IT) solution for the problems identified in the study in order to improve effectiveness or efficiency of knowledge practices. 


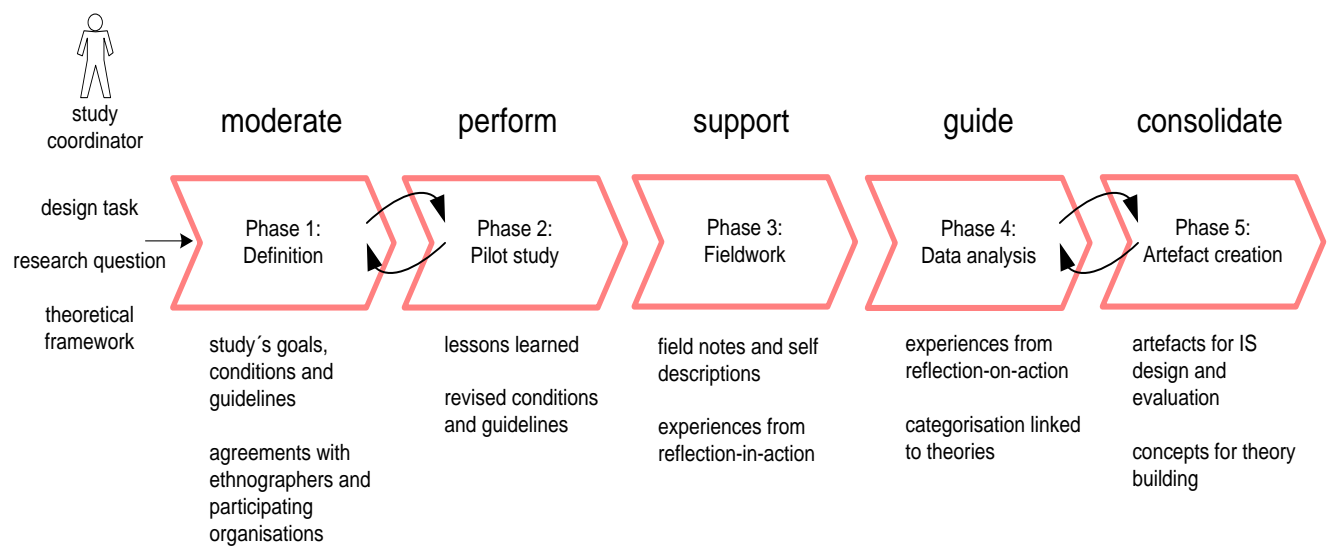

Figure 1: Phases of the procedure

Our approach targets "wicked problems" and supports their analysis and solution with the help of design artefacts (Rittel et al. 1973). Specifically, we use ethnography for investigating ill-defined environmental contexts in order to clearly identify requirements and constraints. Our approach explicitly considers multiple views of participating researchers and their goal-driven consolidation. The conceptual artefacts, such as personas and knowledge processes, created with concepts, theories and subsequent design in mind help to reduce the cognitive load on the designers. Collaboration is strongly encouraged to understand the problem domain in its social environment and renders design a collaborative activity. Together with involving designers in collaborative ethnography, this enhances their social abilities so that resulting solutions are potentially more effective across the investigated social settings.

We identified a critical issue concerning the timing for when the data analysis should be handed over from individual ethnographers to the team of ethnographers. It was difficult for ethnographers to let go of the privilege - and burden - to be the only ones to understand and interpret the specific context of a setting. Coordinating this procedure is necessary, so that the creation of design artefacts benefits from the broader context of several study instances interpreted simultaneously.

Overlapping phases were highly effective with the authors always being the first to perform a step of the study, collect results, present them to ethnographers, agree on the procedure, send out templates, collect filled templates, consolidate them, agree on results and then convey them to ethnographers including designers. Concerning validation of results, strong agreement among ethnographers and strong take-up of artefacts can be seen as internal validation of study results as well as first external validation by designers as customers. Evidence for a strong take-up of artefacts is given by their reuse in richly describing use cases with the help of personas and knowledge practices, reuse and further refinement of the models in the project's model-driven software architecture, and finally, by taking up concepts such as knowledge indicators in the project's evaluation activities.

The study follows a different layout compared to traditional ethnography. The main difference is that time spent for fieldwork was limited to at least four weeks per study instance, with two weeks of ethnographic fieldwork on site. While a longer time frame would have been desirable, this was not feasible due to limited resources on the part of participant organisations which had to agree to open up to ethnographers.

Most study instances built on established relationships so that time required for immersion could be shortened substantially. However, this could also have caused another limitation, namely that ethnographers become socialised to the views of participants and lose the benefit of gaining a fresh 
perspective of the situation (Walsham 2006). In order to account for this, two organisations were chosen without prior relationships to ethnographers, however, their results were in line with other study instances. Our efforts in the study design, guidelines and coordination were important to overcome this potential bias. This also helped to compensate for the fact that individual researchers generally have their own objectives (Hughes et al. 1994). The coordination of a large and diverse team of ethnographers is a major challenge, which we were able to handle with the help of extensive communication, boundary objects and the institutionalised role of a study coordinator.

We also realized that flexibility in applying guidelines and templates is critical. Guidelines and templates should be sufficiently specific to properly guide ethnographers and should allow individual freedom and openness for unknown phenomena. This flexibility demands a multi-perspective procedure in which ethnographers reflect on aspects added by others. Flexibility and number of joint reflection meetings demand time and resources, enhance sensitivity for new aspects and have to be defined case by case.

Summing up, our proposed procedure based on collaborative ethnography offers four advantages over other qualitative methods in general and traditional ethnography in particular, as follows:

1. The context is broadened to include several study instances which are conducted and analysed in a coordinated manner. The variety of multiple social settings as well as the backgrounds and perspectives of many ethnographers could be incorporated (18 in our case). However, this means ethnographers need to collaborate which requires coordination. Our procedure gives a number of recommendations on how to address this non-trivial challenge. Compared to traditional ethnography, advantages of our approach are the broadening of focus and the inclusion of multiple views.

2. The procedure's advantageous time constraints allow investigating organisations not willing to open up for extended periods of time. Compared with interviews or observations, ethnographers can still immerse themselves much more deeply into the social settings and hence are able to gain a greater insight into motivations and intentions. However, the following core of ethnography must be ensured to apply the procedure successfully. Immersion into the field needs to be ensured. Taking our experiences into account, the time spent on site should be divided into at least two one week periods working together with one group of people. Factors reducing the time needed for immersion include previous trusted relationships with subjects, familiarity with the domain and the aspects to be studied as well as similar professional and cultural background as the subjects. Researchers need to participate in the subjects' natural environments to get a real understanding of the happenings. Working together with people in constructed situations or in predefined tasks contradicts the nature of ethnography and limits the ability to understand the happenings. Furthermore, taking part in social interactions needs to be achieved to emphasize the added value compared to observations.

3. The procedure addresses "wicked" problems and informs requirements engineering and IS design. Our procedure enables more time-efficient investigations compared to traditional ethnographies without losing the openness to unforeseen phenomena. Hence, it can be integrated more easily into research and development projects. However, compared with traditional requirements elicitation approaches, e.g. brainstorming, document analysis or interviews (Hossenlopp et al. 2008), more time is needed for the investigation. The larger variety of investigated social settings allows for a comparison across study instances which leads to a heightened level of justification for following design activities. Compared to interviews or observations, the greater variety and richness of study results enhances opportunities for an indepth description of "wicked" problems that can then be addressed by design activities. The increased complexity is influenced by the clarity of the problem, the level of change and the 
number of stakeholders involved (Hossenlopp et al. 2008). Considering that our approach is able to target such highly complex settings, the greater investment in time and effort seems justified. For design tasks that do not address "wicked" problems or focus on incremental improvement, such as the minor further development of existing systems, less time-consuming methods seem more appropriate.

4. Our procedure motivates for involving designers in the study. The early and direct involvement of designers and implementers fosters the take up of study results for the ensuing design activities. It seems beneficial to bring both groups together as early as possible as it helps to align the study's goals with the needs of design. Knowing the designers' and the developers' ideas helps to define the focus areas and can be taken into account by ethnographers during fieldwork. Collaboration between designers and ethnographers also makes it easier to transform study results into design artefacts. This is considered an advantage as study results are usually only handed over from researchers to designers who then create design artefacts independently. Reflecting study results in meetings with ethnographers and designers turned out very beneficial to address the evaluation criteria, e.g. the principle of suspicion (Klein et al. 1999) and transfer first-hand experiences of social settings to designers which can be used as forms of reality check for their ideas and to which they can always turn back.

\section{CONCLUSION}

Traditional ethnography is of limited help in IS design for little researched, complex, dynamic and innovative knowledge work. This paper discussed modified collaborative ethnography for investigating socio-technical organisational settings aimed at designing IS that are useful in these settings. Collaboration broadens the focus, integrates different perspectives and multiplies the time spent on ethnographic fieldwork for investigating the "wicked" problem in case of limited accessibility of organisations. However, it requires coordination mechanisms that balance openness to relevant facets required by ethnography with the focus, structure and results required by IS design. The proposed procedure for modified collaborative ethnography is thus able to provide a much richer picture compared to traditional requirements engineering methods. This can be achieved by considering the social context, problems and solutions that people performing knowledge work can neither easily capture in interviews, nor can they be straightforwardly recognised in observations. This modified approach is faster than traditional ethnographic studies, but requires collaboration, focus and coordination and thus, more time than traditional requirements engineering methods. From a business perspective, the approach should therefore only be applied for innovative, challenging and complex design tasks that are worth the additional effort.

Coordination was aided by a variety of boundary objects and the institutionalised role of a study coordinator. Boundary objects are essential for building new concepts and theories on studied phenomena and for communicating results to designers. The study coordinator drives the procedure, moderates, supports, guides and applies instruments for consolidation and yet must not interfere with the creative processes taking place when ethnographers collect and interpret their rich data.

The paper also reports experiences from a large-scale IS research and development project for which the procedure was operationalised and critically discusses its application and limitations. Our approach, we argue, is especially suited for more innovative, large and diverse study settings, projects, designers, intended IS, and target groups. Artefacts such as personas and processes can reduce the effort for end-users and help keep a consequent focus on how users perform practices of knowledge work. Although our approach requires much less time compared to traditional ethnography, the effort of carefully preparing and coordinating a collaborative study should not be underestimated and needs still more time than traditional requirements elicitation methods. 
Avenues for future work are to integrate more closely our procedure with approaches to user-centred design. In extension of users bringing on board their explicit knowledge limited to what they are aware of and deem important for the design problem at hand, ethnography taps into the potential of understanding what users actually do, how they do it and why they do it in a much more profound way. Another area worth further research is the analysis of factors which determine the right balance between the added effort for modified collaborative ethnography in IS design and the superiority of the resulting IT-supported knowledge work practices. Finally and from an academic perspective, it seems promising to continue the refinement of research methods that help build a bridge between (behavioural) computer science and qualitative-interpretive as well as design science-oriented IS research communities.

\section{ACKNOWLEDGEMENTS}

This work was co-funded by the European Commission under the Information and Communication Technologies theme of the $7^{\text {th }}$ Framework Programme, Integrating Project MATURE (Contract No. 216356, http://mature-ip.eu).

We thank the reviewers for the insightful and constructive comments that helped improve the paper substantially.

\section{REFERENCES}

Agar, M. H. The Professional Stranger: An Informal Introduction to Ethnography, Academic Press, New York, NY, 1980.

Al-Karaghouli, W., Alshawi, S., and Fitzgerald, G. "Promoting Requirement Identification Quality Enhancing the Human Interaction Dimension," Journal of Enterprise Information Management (18:2) 2005, pp 256-267.

Alavi, M., and Leidner, D. E. "Review: Knowledge Management and Knowledge Management Systems: Conceptual Foundations and Research Issues," Management Information Systems Quarterly (25:1) 2001, pp 107-136.

Alvesson, M. Knowledge Work and Knowledge-Intensive Firms, Oxford, 2004.

Atkinson, P., and Hammersley, M. Ethnography - Principles in Practice, (3rd ed.) Routledge, New York, 2007.

Baird , L., Holland, P., and Deacon, S. "Learning from Action: Imbedding More Learning into the Performance Fast Enough to Make a Difference," Organizational Dynamics (27:4) 1999, pp 19-32.

Ball, L. J., and Ormerod, T. C. "Applying Ethnography in the Analysis and Support of Expertise in Engineering Design," Design Studies (21:4) 2000, pp 403-421.

Barley, S. R., and Kunda, G. "Bringing Work Back In," Organization Science (12:1) 2001, pp 76-95.

Benbasat, I., Goldstein, D. K., and Mead, M. "The Case Research Strategy in Studies of Information Systems," Management Information Systems Quarterly (11:1) 1987, pp 369-386.

Benbasat, I., and Zmud, R. W. "Empirical Research in Information Systems: The Practice of Relevance," Management Information Systems Quarterly (23:1) 1999, pp 3-16. 
Bentley, R., Hughes, J. A., Randall, D., Rodden, T., Sawyer, P., Shapiro, D., and Sommerville, I. "Ethnographically-Informed Systems Design for Air Traffic Control," ACM Conference on Computer-Supported Cooperative Work, ACM, Toronto, Canada 1992, pp. 123-129.

Bishop, J., Bouchlaghem, D., Glass, J., and Matsumoto, I. "Ensuring the Effectiveness of a Knowledge Management Initiative," Journal of Knowledge Management (12:4) 2008, pp 1629.

Blackler, F. "Knowledge, Knowledge Work and Organizations: An Overview and Interpretation," Organization Studies (16:6) 1995, pp 1021-1046.

Blackler, F., Reed, M., and Whitaker, A. "Epilogue - An Agenda for Research," Journal of Management Studies (30:6) 1993, pp 1017-1020.

Brown, J. S., and Duguid, P. "Organizing Knowledge," California Management Review (40:3) 1998, pp 90-111.

Buford-May, R. A. B., and Pattillo-McCoy, M. "Do You See What I See? Examining a Collaborative Ethnography," Qualitative Inquiry (6:1) 2001, pp 65-87.

Chang, Y., Lim, Y., and Stolterman, E. "Personas: From Theory to Practices," Proceedings of the $5^{\text {th }}$ Nordic Conference on Human-Computer Interaction: Building Bridges, ACM, Lund, Sweden, 2008, pp. 439-442.

Chatterjee, D., Grewal, R., and Sambamurthy, V. "Shaping up for E-Commerce: Institutional Enablers of the Organizational Assimilation of Web Technologies," Management Information Systems Quarterly (26:2) 2002, pp 65-89.

Cohen, A., and Court, D. "Ethnography and Case Study: A Comparative Analysis," Academic Exchange Quarterly 2003.

Crabtree, A. Designing Collaborative Systems - A Practical Guide to Ethnography, Springer, London, 2003.

Creswell, J. W. Research Design: Qualitative, Quantitative, and Mixed Method Approaches, (2nd ed.) SAGE, Thousand Oaks, 2003.

Darke, P., Shanks, G., and Broadbent, M. "Successfully Completing Case Study Research: Combining Rigour, Relevance and Pragmatism," Information Systems Journal (8:4) 2002, pp 273-289.

Davenport, T. H. "Putting the Enterprise into the Enterprise System," Harvard Business Review (76:4) 1998, pp 121-131.

Davenport, T. H., Jarvenpaa, S. L., and Beers, M. C. "Improving Knowledge Work Processes," Sloan Management Review (37:4) 1996, pp 53-65.

Davis, A. M., Hull, S. C., Grady, C., Wilfond, B. S., and Henderson, G. E. "The Invisible Hand in Clinical Research: The Study Coordinator's Critical Role in Human Subjects Protection," Journal of Law, Medicine \& Ethics (30:3) 2002, pp 411-419.

Delone, W. H., and McLean, E. R. "The DeLone and McLean Model of Information Systems Success: A Ten-Year Update," Journal of Management Information Systems (19:4) 2003, pp 9-30.

Dennis, A. R. "Information Exchange and Use in Group Decision Making: You Can Lead a Group to Information, but You Can't Make It Think.," Management Information Systems Quarterly (20:4) 1996, pp 433-455. 
Dickover, N. "Reflection-in-Action: Modeling a Specific Organization Through the Viable Systems Model," Systems Practice (7:1) 1994, pp 43-62.

Dillon, A., and Morris, M. "User Acceptance of Information Technology: Theories and Models," Annual Review of Information Science and Technology (31) 1996.

Drucker, P. F. Landmarks of Tomorrow, Harper, New York, 1959.

Drucker, P. F. "The Age of Social Transformation," The Atlantic Monthly (274:5) 1994, pp 53-80.

Dufon, M. A. "Video Recording in Ethnographic SLA Research: Some Issues of Validity in Data Collection," Language Learning \& Technology (6:1) 2002, pp 40-59.

Ellis, D. "Modeling the Information-Seeking Patterns of Academic Researchers - A Grounded Theory Approach," Library Quarterly (63:4) 1993, pp 469-486.

Emmerson, R. M., Fretz, R. I., and Shaw, L. L. Writing Ethnographic Field Notes, University of Chicago Press, Chicago, 1995.

Fetterman, D. M. Ethnography Step by Step, (2nd ed.) Sage, London, 1999.

Fetterman, D. M. Ethnography Step-by-Step, (3rd Ed.) Sage, Thousand Oaks, 2010.

Glaser, B., and Strauss, A. The Discovery of Grounded Theory: Strategies for Qualitative Research, Aldine, Chicago, 1967.

Glaser, B. G., and Strauss, A. L. "Die Entdeckung gegenstandsbezogener Theorie: Eine Grundstrategie qualitativer Sozialforschung," Hopf, C. and Weingarten, E.H. (eds.), Qualitative Sozialforschung, Klett, Stuttgart, 1979, pp. 91-111.

Gläser, J., and Laudel, G. Experteninterviews und qualitative Inhaltsanalyse, (2nd ed.) Verlag für Sozialwissenschaften Wiesbaden, 2006.

Goodhue, D. L., and Thompson, R. L. "Task-Technology Fit and Individual Performance.," Management Information Systems Quarterly (19:2), pp 213-236.

Gordon, T., Hynninen, P., Lahelma, E., Metso, T., Palmu, T., and Tolonen, T. "Collective Ethnography, Joint Experiences and Individual Pathways," Nordisk Pedagogik (26:1) 2006, pp $3-15$.

Graham, P., Bowerman, C., and Bokma, A. "Adaptive Navigation for Mobile Devices," . Attewell, J. and Savill-Smith, C. (eds.), Learning with Mobile Devices Research and Development, Learning and Skills Development Agency, London, 2004, pp. 61-68.

Hamilton, S., and Chervany, N. L. "Evaluating Information System Effectiveness - Part II: Comparing Evaluator Viewpoints," Management Information Systems Quarterly (5:4) 1981, pp 79-86.

Hammersley, M., and Atkinson, P. Ethnography Principles in Practice, (3rd ed.) Routledge, 2007.

Harper, R. H. R. "The Organisation in Ethnography: A Discussion of Ethnographic Fieldwork Programs in CSCW," Computer Supported Cooperative Work (9:2) 2000, pp 239-264.

Hayes, N. "Boundless and Bounded Interactions in the Knowledge Work Process: The Role of Groupware Technologies," Information and Organization (11:2) 2001, pp 79-101.

Hevner, A. R., March, S. T., Park, J., and Ram, S. "Design Science in Information Systems Research," Management Information Systems Quarterly (28:1) 2004, pp 75-105.

Hine, C. Virtual Ethnography, Sage, London, 2000. 
Horsburgh, D. "Evaluation of Qualitative Research," Journal of Clinical Nursing (12:2) 2003, pp 307312.

Hossenlopp, R., and Hass, K. B. Unearthing Business Requirements: Elicitation Tools and Techniques, Management Concepts, Vienna, VA, USA, 2008.

Hughes, J. A., King, V., Rodden, T., and Andersen, H. "Moving out of the Control Room: Ethnography in Systems Design.," ACM Conference on Computer Supported Cooperative Work, ACM, Chapel Hill, USA 1994, pp. 429 - 439.

Jordan, B. "Ethnographic Workplace Studies and CSCW," Shapiro, D., Tauber, M., and Traunmüller, R. (eds.) The Design of Computer Supported Cooperative Work and Groupware Systems, Elsevier, Amsterdam, Netherlands, 1996, pp. 17-42.

Kaplan, B., and Duchon, D. "Combining Qualitative and Quantitative Methods in Information Systems Research: A Case Study," Management Information Systems Quarterly (12:4) 1988, pp 571-586.

Kelloway, E. K., and Barling, J. "Knowledge Work as Organizational Behavior," International Journal of Management Reviews (2:3) 2000, pp 287-304.

Kendall, K. E., and Losee, R. D. "Information System FOLKLORE: A New Technique for System Documentation," Information \& Management (10:2) 1986, pp 103-111.

Klein, H., and Myers, M. D. "A Set of Principles for Conducting and Evaluating Interpretive Field Studies in Information Systems," Management Information Systems Quarterly (23:1) 1999, pp 67-94.

Knights, D., Murray, F., and Willmott, H. "Networking as Knowledge Work: A Study of Strategic Interorganizational Development in the Financial Services Industry," Journal of Management Studies (30:6) 1993, pp 975-995.

Lassiter, L. E. "Collaborative Ethnography and Public Anthropology," Current Anthropology (46:1) 2005, pp 83-105.

Leigh Star, S., and Griesemer, J. R. "'Translations' and Boundary Objects: Amateurs and Professionals in Berkeley's Museum of Vertebrate Zoology," Social Studies of Science (19:3) 1989, pp 387-420.

Lewis, S., Mateas, M., Palmiter, S., and Lynch, G. "Ethnographic Data for Product Development: A Collaborative Process," Interactions (3:6) 1996, pp 52-69.

Lincoln, Y. S. "Emerging Criteria for Quality in Qualitative and Interpretive Research," Qualitative Inquiry (1:3) 1995, pp 275-289.

Luna-Reyes, L. F., Black, L. J., Cresswell, A. M., and Pardo, T. A. "Knowledge Sharing and Trust in Collaborative Requirements Analysis," System Dynamics Review (24:3) 2008, pp 265-297.

Maginn, P. J. "Towards More Effective Community Participation in Urban Regeneration: The Potential of Collaborative Planning and Applied Ethnography," Qualitative Research (7:1) 2007, pp 25-43.

Maier, R. "Modeling Knowledge Work for the Design of Knowledge Infrastructures," Journal of Universal Computer Science (11:4) 2005, pp 429-451.

Malinowski, B. Argonauts of the Western Pacific, Dutton \& Co. Inc., New York, 1922. 
Malone, T. W., and Crowston, K. "The Interdisciplinary Study of Coordination," ACM Computing Surveys (26:1) 1994, pp 87-119.

Markus, M. L., Majchrzak, A., and Gasser, L. "A Design Theory for Systems that Support Emergent Knowledge Processes," Management Information Systems Quarterly (26:3) 2002, pp 179-212.

Millen, D. R. "Rapid Ethnography: Time Deepening Strategies for HCI Field Research," Conference on Designing Interactive Systems, ACM Press, New York, USA, 2000, pp. 280-286.

Myers, M. "Investigating Information Systems with Ethnographic Research," Communications of the Association for Information Systems (2:4) 1999, pp 1-22.

Myers, M. D., and Young, L. W. "Hidden Agendas, Power, and Managerial Assumptions in Information Systems Development: An Ethnographic Study," Information Technology \& People (10:3) 1997, pp 224-240.

Nonaka, I. "The Knowledge-Creating Company," Harvard Business Review (69:6) 1991, pp 96-104.

O'Dell, C., and Grayson, C. J. "If We Only Knew What We Know: Identification and Transfer of Internal Best Practices," California Management Review (40:3) 1998, pp 154-174.

Orlikowski, W. J. "Integrated Information Environment or Matrix of Control? The Contradictory Implications of Information Technology," Accounting, Management and Information Technologies (1:1) 1991, pp 9-42.

Orlikowski, W. J. "CASE Tools as Organizational Change: Investigating Incremental and Radical Changes in Systems Development," Management Information Systems Quarterly (17:3) 1993, pp 309-340.

Patton, M. Q. Qualitative Research \& Evaluation Methods, (3rd ed.) Sage, Thousand Oaks, 2002.

Penrose, E. The Theory of the Growth of the Firm, John Wiley and Sons, New York, 1959.

Pink, S. Doing Visual Ethnography: Images, Media and Representation in Research, (2nd ed.) Sage, London, 2006.

Plowman, L., Rogers, Y., and Ramage, M. "What Are Workplace Studies For?," 4th European Conference on Computer-Supported Cooperative Work, Stockholm, Sweden, 1995, pp. 309 324.

Pruitt, J., and Adlin, T. The Persona Lifecycle, Keeping People in Mind Throughout Product Design, Kaufmann Publisher, San Francisco, 2006.

Rittel, H. W. J., and Webber, M. M. "Dilemmas in a General Theory of Planning," Policy Sciences (4:2) 1973, pp 155-169.

Robinson, H., Segal, J., and Sharp, H. "Ethnographically-Informed Empirical Studies of Software Practice," Information and Software Technology (49:6) 2007, pp 540-551.

Rogers, E. Diffusion of Innovations, Free Press, New York, 1995.

Schön, D. A. The Reflective Practitioner, Basic Books, 1983.

Schultze, U. "A Confessional Account of an Ethnography About Knowledge Work," Management Information Systems Quarterly (24:1) 2000, pp 3-41.

Schultze, U. "On Knowledge Work," Holsapple, C.W. (ed.), Handbook on Knowledge Management, Springer, Berlin, 2003, pp. 43-58.

Seale, C. "Quality in Qualitative Research," Qualitative Inquiry (5:4) 1999, pp 465-478. 
Sharrock, W., and Randall, D. "Ethnography, Ethnomethodology and the Problem of Generalisation in Design," European Journal of Information Systems (13:3) 2004, pp 186-194.

Simonsen, J., and Kensing, F. "Using Ethnography In Contextual Design," Communications of the Association for Information Systems (40:7) 1997, pp 82-88.

Starbuck, W. H. "Learning by Knowledge-Intensive Firms," Journal of Management Studies (29:6) 1992, pp 713-740.

Strauss, A., and Corbin, J. Basics of Qualitative Research: Grounded Theory, Procedures, and Techniques, Sage, Newbury Park, CA, USA, 1990.

Suchman, L. Plans and Situated Actions: The Problem of Human-Machine Communication, Cambridge University Press, New York, 1987.

Suchman, L. "Making Work Visible," Communications of the ACM (38:9) 1995, pp 56-64.

Tuula, G., Hynninen, P., Lahelma, E., Metso, T., Palmu, T., and Tolonen, T. "Collective Ethnography, Joint Experiences and Individual Pathways," Nordisk Pedagogik (26:1) 2006, pp 3-15.

Viller, S., and Sommerville, I. "Ethnographically Informed Analysis for Software Engineers," International Journal of Human-Computer Studies (53:1) 2000, pp 169-196.

Visconti, L. M. "Ethnographic Case Study (ECS): Abductive Modeling of Ethnography and Improving the Relevance in Business Marketing Research," Industrial Marketing Management (39:1) 2010, pp 25-39.

Walsham, G. "Interpretive Case Studies in IS Research: Nature and Method," European Journal of Information Systems (4:2) 1995, pp 74-81.

Walsham, G. "Doing Interpretive Research," European Journal of Information Systems (15:3) 2006, pp 320-330.

Warne, L., Pascoe, C., and Ali, I. "Painting With and Without Numbers: The Use of Qualitative and Quantitative Methods to Study Social Learning," Australasian Journal of Information Systems (12:2) 2005, pp 112-120.

Weng, C., McDonald, D. W., Sparks, D., McCoy, J., and Gennari, J. H. "Participatory Design of a Collaborative Clinical Trial Protocol Writing System," International Journal of Medical Informatics (76:1) 2006, pp S245-S251.

White, J., Drew, S., and Hay, T. "Ethnography Versus Case Study," Qualitative Research Journal (9:1) 2009, pp 18-27.

Whitehead, J. "Collaboration in Software Engineering: A Roadmap " Briand, L. C., Wolf, A. L. (eds.) Future of Software Engineering 2007, Minneapolis, Minnesota, pp 214-225.

Wiig, K. M. Knowledge Management Foundations. Thinking about Thinking. How People and Organizations Create, Represent, and Use Knowledge, Schema Press, Arlington (TX, USA), 1993.

Wolff, E. N. "The Growth of Information Workers," Communications of the ACM (48:10) 2005, pp $37-42$.

Wu, J., Graham, T. C. N., and Smith, P. W. "A Study of Collaboration in Software Design," ISESE '03 International Symposium on Empirical Software Engineering IEEE, Rome, Italy, 2003.

Zack, M. H. "Managing Codified Knowledge.," Sloan Management Review (40:4) 1999, pp 45-58. 